\title{
Electronic Interaction Integrals for Atoms Calculated with Laguerre Polynomial Radial Wavefunctions*
}

\author{
ROBERT T. BROWN $\dagger$ \\ Department of Physics, The University of Michigan, Ann Arbor, Michigan
}

(Received 29 August 1966)

\begin{abstract}
Straightforward evaluation of electronic interaction integrals for many-electron atoms using Laguerre polynomial radial wavefunctions leads to sums of differences of large numbers. An alternate formulation is presented which does not have this disadvantage.
\end{abstract}

$\mathbf{A}$ COMPLETE set of functions which has come into increased use in recent years as an expansion basis in variational calculations of atomic energy states is the set of Laguerre polynomials which differ from hydrogenic functions in that the principal quantum number $n$ does not appear in the argument. ${ }^{1-6}$ These functions have the important advantages over hydrogenic functions that they form a complete denumerable set, with no continuum states, ${ }^{7}$ and that the nodes do not move out from the origin with increasing $n$. This second feature leads to improved convergence since the nodes remain closer to the region in which the function to be represented has its most interesting features. ${ }^{8}$
It is the purpose of this article to point out a property of matrix elements calculated using these functions which could lead to rapid loss of precision in numerical computations and to indicate a way in which this difficulty can be circumvented.

The radial function is

$$
R_{n l}(r)=\frac{[\Gamma(n-l)]^{1 / 2}}{[\Gamma(n+l+1)]^{3 / 2}}(2 k r)^{l} \exp (-k r) L_{n-l-1}{ }^{2 l+1}(2 k r),
$$

where $L_{n-l-1}{ }^{2 l+1}(2 k r)$ can be written in terms of the confluent hypergeometric series

$$
L_{a}^{b}(\xi)=\frac{[\Gamma(a+b+1)]^{2}}{\Gamma(a+1) \Gamma(b+1)} \sum_{j} \frac{\Gamma(-a+j) \Gamma(b)}{\Gamma(-a) \Gamma(b+j)} \frac{\xi^{j}}{j !} .
$$

The matrix element of the interelectron Coulomb repulsion contains the radial integral

$$
\begin{aligned}
\left(n_{1} l_{1} n_{2} l_{2}\left|\frac{\xi^{\lambda}}{\xi^{\lambda+1}}\right| n_{3} l_{3} n_{4} l_{4}\right)=\int_{0}^{\infty} d \eta\left[\int_{0}^{\eta} d \rho R_{n_{1} l_{1}}(\rho) \rho^{\lambda+2} R_{n_{3} l_{3}}(\rho)\right] R_{n_{2} l_{3}}(\eta) \eta^{1-\lambda} R_{n_{4} l_{4}}(\eta) & \\
& +\int_{0}^{\infty} d \eta\left[\int_{0}^{\eta} d \rho R_{n_{2} l_{2}}(\rho) \rho^{\lambda+2} R_{n_{4} l_{4}}(\rho)\right] R_{n_{1} l_{3}}(\eta) \eta^{1-\lambda} R_{n_{3} l_{3}}(\eta),
\end{aligned}
$$

where $\xi=2 k r, \rho=\xi_{<}$, and $\eta=\xi$. Straightforward evaluation of the integral (3), hereafter denoted $I$, gives the result

$$
\begin{aligned}
& I=\left[\frac{\left(n_{1}+l_{1}\right) !\left(n_{2}+l_{2}\right) !\left(n_{3}+l_{3}\right) !\left(n_{4}+l_{4}\right) !}{\left(n_{1}-l_{1}-1\right) !\left(n_{2}-l_{2}-1\right) !\left(n_{3}-l_{3}-1\right) !\left(n_{4}-l_{4}-1\right) !}\right]^{1 / 2} \\
& \times \sum_{\mu, \nu} \sum_{\sigma, \tau} \frac{(-1)^{\mu+\nu+\sigma+\tau}\left(\begin{array}{c}
n_{1}-l_{1}-1 \\
\mu
\end{array}\right)\left(\begin{array}{c}
n_{2}-l_{2}-1 \\
\sigma
\end{array}\right)\left(\begin{array}{c}
n_{3}-l_{3}-1 \\
\nu
\end{array}\right)\left(\begin{array}{c}
n_{4}-l_{4}-1 \\
\tau
\end{array}\right)}{2^{l_{1}+l_{2}+l_{3}+l_{4}+\mu+\nu+\sigma+\tau+4}\left(2 l_{1}+1+\mu\right) !\left(2 l_{2}+1+\sigma\right) !\left(2 l_{3}+1+\nu\right) !\left(2 l_{4}+1+\tau\right) !} \\
& \times\left[\left(l_{2}+l_{4}+\sigma+\tau-\lambda+1\right) !\left(l_{1}+l_{3}+\mu+\nu+\lambda+2\right) ! \sum_{j=0}^{l_{2}+l_{4}+\sigma+\tau-\lambda+1}\left(\begin{array}{c}
l_{1}+l_{2}+l_{3}+l_{4}+\mu+\nu+\sigma+\tau+4 \\
j
\end{array}\right)\right. \\
& \left.+\left(l_{1}+l_{3}+\mu+\nu-\lambda+1\right) !\left(l_{2}+l_{4}+\sigma+\tau+\lambda+2\right) ! \sum_{j=0}^{l_{3}+l_{3}+\mu+\nu-\lambda+1}\left(\begin{array}{c}
l_{1}+l_{2}+l_{3}+l_{4}+\mu+\nu+\sigma+\tau+4 \\
j
\end{array}\right)\right],
\end{aligned}
$$

* This work was supported in part by the U.S. Atomic Energy Commission under Contract AT(11-1)-1112.

$\dagger$ Present address: Department of Space Science, Rice University, Houston, Texas.

1 P..O. Löwdin and H. Shull, Phys. Rev. 101, 1730 (1956).

2 D. H. Tycko, L. H. Thomas, and K. M. King, Phys. Rev. 109, 369 (1958).

${ }^{3}$ R. K. Nesbet and R. E. Watson, Phys. Rev. 110, 1073 (1958).

4 H. Shull and P.-O. Löwdin, J. Chem. Phys. 30, 617 (1959).

${ }^{5}$ H. L. Davis, J. Chem. Phys. 39, 1827 (1963).

- R. T. Brown and P. R. Fontana, J. Chem. Phys. 45, 4248 (1966).

${ }^{7}$ M. Rotenberg, Ann. Phys. (N.Y.) 14, 262 (1962).

${ }^{8}$ J. C. Slater, Quantum Theory of Atomic Structure (McGraw-Hill Book Co., New York, 1960), Vol. 2, p. 45. 
where the range of summation is limited by the range of definition of the binomial coefficients. This would now appear to be in an ideal form for computation, since all sums are finite and all variables are integers. However, in practice it has been found that the alternating signs of the terms in the quadruple sum quickly lead to loss of precision, since the magnitude of the sum is in general smaller than the magnitudes of the individual terms, with the difference in size growing rapidly with increasing $n_{i}$.

A solution to this difficulty can be found by rewriting the quadruple sum as two double sums, one of which can be expressed as the generalized hypergeometric function of two variables ${ }^{9}$ :

$F_{2}\left(a, b, b^{\prime}, c, c^{\prime}, x, y\right)=\sum_{m, n} \frac{(a)_{m+n}(b)_{m}\left(b^{\prime}\right)_{n} x^{m} y^{n}}{(c)_{m}\left(c^{\prime}\right)_{n} m ! n !}$, in which $(a)_{n}=a(a+1) \cdots(a+n-1)=\Gamma(a+n) / \Gamma(a)$. There is then left a double sum of terms which still have alternating signs, but of such magnitude that there is no longer any loss of precision in calculating the sum. In order to reduce the quadruple sum to a double sum the identities

$$
\begin{aligned}
\sum_{j=0}\left(\begin{array}{c}
a+b+1 \\
j
\end{array}\right) & =2^{a+b+1}-\sum_{j=0}^{b}\left(\begin{array}{c}
a+b+1 \\
j
\end{array}\right) \\
& =2^{a} \sum_{j=0}^{a}\left(\begin{array}{c}
b+j \\
j
\end{array}\right) \frac{1}{2^{j}},
\end{aligned}
$$

are applied to Eq. (4) and the binomial coefficients rewritten by means of the relationships

$$
\left(\begin{array}{l}
a \\
n
\end{array}\right)=\frac{\Gamma(a+1)}{\Gamma(n+1) \Gamma(a-n+1)}=\frac{(-1)^{n} \Gamma(-a+n)}{\Gamma(n+1) \Gamma(-a)}=(-1)^{n} \frac{(-a)_{n}}{n !} .
$$

Note that this makes it possible to remove either $\sigma$ and $\tau$ or $\mu$ and $\nu$ as variables in the truncated binomial series. The choice is made so that the summation indices with wider range are removed, in order to minimize as far as possible the error caused by the alternating signs. For the case $n_{1}-l_{1}+n_{3}-l_{3}<n_{2}-l_{2}+n_{4}-l_{4}$, the result is

$$
\begin{gathered}
I=\left[\frac{\left(n_{1}+l_{1}\right) !\left(n_{2}+l_{2}\right) !\left(n_{3}+l_{3}\right) !\left(n_{4}+l_{4}\right) !}{\left(n_{1}-l_{1}-1\right) !\left(n_{2}-l_{2}-1\right) !\left(n_{3}-l_{3}-1\right) !\left(n_{4}-l_{4}-1\right) !}\right]^{1 / 2}\left\{\frac{\left(l_{1}+l_{3}+\lambda+2\right) !\left(l_{2}+l_{4}-\lambda+1\right) !}{\left(2 l_{1}+1\right) !\left(2 l_{2}+1\right) !\left(2 l_{3}+1\right) !\left(2 l_{4}+1\right) !}\right. \\
\times F_{2}\left(l_{1}+l_{3}+\lambda+3,-n_{1}+l_{1}+1,-n_{3}+l_{3}+1,2 l_{1}+2,2 l_{3}+2,1,1\right) \\
\times F_{2}\left(l_{2}+l_{4}-\lambda+2,-n_{2}+l_{2}+1,-n_{4}+l_{4}+1,2 l_{2}+2,2 l_{4}+2,1,1\right) \\
+\frac{1}{2^{l_{2}+l_{4}+4}} \sum_{\mu, \nu}(-1)^{\mu+\nu}\left[\left(\begin{array}{c}
n_{1}-l_{1}-1 \\
\mu
\end{array}\right)\left(\begin{array}{c}
n_{3}-l_{3}-1 \\
\nu
\end{array}\right) /\left(2 l_{1}+1+\mu\right) !\left(2 l_{3}+1+\nu\right) !\right] \\
\times\left[\left(\mu+\nu+l_{1}+l_{3}-\lambda+1\right) ! \sum_{j=0}^{\mu+\nu+l_{1}+l_{8}-\lambda+1} \frac{\left(l_{2}+l_{4}+\lambda+2+j\right) !}{j ! 2^{\lambda+j-1}}\right. \\
\times F_{2}\left(l_{2}+l_{4}+\lambda+3+j,-n_{2}+l_{2}+1,-n_{4}+l_{4}+1,2 l_{2}+2,2 l_{4}+2, \frac{1}{2}, \frac{1}{2}\right) \\
-\left(\mu+\nu+l_{1}+l_{3}+\lambda+2\right) ! \sum_{j=0}^{\mu+\nu+l_{1+1}+\lambda+2} \frac{\left(l_{2}+l_{4}-\lambda+1+j\right) !}{j ! 2^{j-\lambda-2}} \\
\left.\left.\times F_{2}\left(l_{2}+l_{4}-\lambda+2+j,-n_{2}+l_{2}+1,-n_{4}+l_{4}+1,2 l_{2}+2,2 l_{4}+2, \frac{1}{2}, \frac{1}{2}\right)\right]\right\},
\end{gathered}
$$

with a corresponding expression for the other possibility for the relative magnitudes of the limits on the sums. This result is actually simpler than Eq. (4), because the generalized hypergeometric (5) is a single sum when the two variables are equal. It has been found that with this rearrangement of the summation, single precision arithmetic ( 27 bit mantissa) is adequate for handling terms with $n \leq 24$, whereas with the original form (4) double precision arithmetic becomes necessary at $n=10$, and inadequate at $n=16$.

A. Erdélyi, W. Magnus, F. Oberhettinger, and F. G. Tricomi, Higher Transcendental Functions, Bateman Manuscript Project (McGraw-Hill Book Co., New York, 1952), Vol. 1, p. 224. 